\title{
Bacterial cell factories for recombinant protein production; expanding the catalogue
}

\author{
Neus Ferrer-Miralles ${ }^{1,2,3}$ and Antonio Villaverde ${ }^{1,2,3^{*}}$
}

Escherichia coli has been the pioneering host for recombinant protein production, since the original recombinant DNA procedures were developed using its genetic material and infecting bacteriophages. As a consequence, and because of the accumulated know-how on $E$. coli genetics and physiology and the increasing number of tools for genetic engineering adapted to this bacterium, E. coli is the preferred host when attempting the production of a new protein. Also, it is still the first choice for protein production at laboratory and industrial scales for an important number of proteins, being fast growth and simple culture procedures critical issues. When searching for an ideal system for protein production, this bacterial species is clearly far from offering, in generic terms, optimal conditions for protein production and downstream. Plasmid loss and antibiotic-based maintenance, undesired chemical inducers of gene expression, plasmid/ protein-mediated metabolic burden and stress responses, lack of post-translational modifications (including the inability to form disulphide bonds), none or poor secretion, protein aggregation and proteolytic digestion, endotoxin contamination and complex downstream are among the main obstacles encountered during protein production in E. coli. In the pharmaceutical scenario, proper protein glycosylation is often requested and simplest purification procedures become highly desirable when pursuing cost-effective bioproduction. In this context, the yeast Sacharomyces cerevisae, diverse mammalian cell lines, insect cells and whole plant and animals (as transgenic systems) are being incorporated to the protein production scenario [1], and many of these products have been already approved for use as protein drugs [2]. Other (less conventional) yeast species and a more limited number of species of filamentous fungi [3], molds [4], moss [5], algae [6] and protozoa [7] are also under development as

\footnotetext{
*Correspondence: antoni.villaverde@uab.cat

'Institut de Biotecnologia i de Biomedicina, Universitat Autònoma de

Barcelona, Bellaterra 08193 Barcelona, Spain

${ }^{2}$ CIBER de Bioingeniería, Biomateriales y Nanomedicina (CIBER-BBN), Bellaterra

08193 Barcelona, Spain

Full list of author information is available at the end of the article
}

potential suppliers of recombinant proteins. The engineering of such systems could represent a promising way to the cost effective production of high quality protein versions that biotechnology and biomedical industries are steadily demanding. The potential and versatility of these platforms as protein producers or in general, as cell factories for added value products such as chemicals, amino acids or vitamins has been stressed in recent experimental reports or reviews [8-17]. Despite this, it must be noted that adapting large-scale production processes to the biological complexity of some of these systems might represent, in some cases, an unaffordable task.

From a different angle, bacterial hosts others than E. coli are attracting attention as cell factories due to their metabolic diversity and biosynthetic potential derived from adaptation to extremely diverse environments. The most important bacterial groups explored as cell factories for recombinant proteins and their associated potentialities are summarized in Table 1. The implementation of lactic acid bacteria as a routine cell factory expands their applications from conventional food microbiology [18-21] to protein production and also protein drug display and delivery [22-29], taking advantage of the generically recognized as safe (GRAS) features of this platform. Improved solubility in halophillic and cold-adapted bacteria, enhanced secretion in acid lactic bacteria and in general in endotoxin-free gram-positive species and post-translational modifications in mycobacteria among others are highly appealing properties in protein production, that can be of special value for specific difficult-to-express proteins. While exhibiting most of the above mentioned limitations linked to prokaryotic-based production, exploring bacterial species other than E. coli should be not abandoned but fully supported as it will not only expand the current catalogue of cell factories but also offer novel process opportunities in easily cultivable/scalable systems that might pose, in generic terms, less methodological issues than unconventional protein production systems [30].

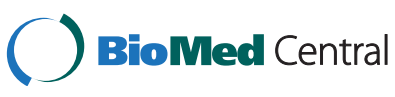

(C) 2013 Ferrer-Miralles and Villaverde; licensee BioMed Central Ltd. This is an Open Access article distributed under the terms of the Creative Commons Attribution License (http://creativecommons.org/licenses/by/2.0), which permits unrestricted use distribution, and reproduction in any medium, provided the original work is properly cited. The Creative Commons Public Domain Dedication waiver (http://creativecommons.org/publicdomain/zero/1.0/) applies to the data made available in this article, unless otherwise stated. 
Table 1 The most important bacterial groups explored as cell factories for recombinant protein production

\begin{tabular}{|c|c|c|c|c|c|c|}
\hline & Host & Main features & Reviews $^{\mathrm{a}}$ & Main bacterial Species & Case proteins & References \\
\hline \multirow[t]{11}{*}{ Proteobacteria } & Caulobacteria & $\begin{array}{l}\text { Easy purification of } \\
\text { secreted RSaA fusions }\end{array}$ & {$[31,32]$} & Caulobacter crescentus & $\begin{array}{l}\text { Hematopoietic necrosis virus } \\
\text { capsid proteins }\end{array}$ & {$[33]$} \\
\hline & & & & & $\beta-1,4-$ glycanase & {$[34]$} \\
\hline & $\begin{array}{l}\text { Phototrophic } \\
\text { bacteria }\end{array}$ & $\begin{array}{l}\text { High production of } \\
\text { membrane proteins }\end{array}$ & {$[35]$} & Rodhobacter sphaeroides & Membrane proteins & {$[35]$} \\
\hline & $\begin{array}{l}\text { Cold adapted } \\
\text { bacteria }\end{array}$ & Improved protein folding & {$[36,37]$} & $\begin{array}{l}\text { Pseudoalteromonas } \\
\text { haloplanktis }\end{array}$ & 3H6 Fab & {$[38]$} \\
\hline & & & & & Human nerve growth factor & {$[39]$} \\
\hline & & & & $\begin{array}{l}\text { Shewanella sp. strain } \\
\text { Ac10 }\end{array}$ & $\beta$-Lactamase, peptidases, glucosidase & {$[40]$} \\
\hline & Pseudomonads & Efficient secretion & [41] & $\begin{array}{l}\text { Pseudomonas } \\
\text { fluorescens }\end{array}$ & $\begin{array}{l}\text { Human granulocyte colony- } \\
\text { stimulating factor }\end{array}$ & {$[42]$} \\
\hline & & & & Pseudomonas putida & Single chain Fv fragments & [43] \\
\hline & & & & $\begin{array}{l}\text { Pseudomonas } \\
\text { aeruginosa }\end{array}$ & Penicillin $\mathrm{G}$ acylase & [44] \\
\hline & $\begin{array}{l}\text { Halophilic } \\
\text { bacteria }\end{array}$ & Solubility favored & {$[45]$} & Halomonas elongata & $\beta$-Lactamase & {$[45]$} \\
\hline & & & & $\begin{array}{c}\text { Chromohalobacter } \\
\text { salexigens }\end{array}$ & Nucleoside diphosphate kinase & {$[46]$} \\
\hline \multirow[t]{8}{*}{ Actinobacteria } & Streptomycetes & Efficient secretion & {$[47,48]$} & Streptomyces lividans & M. tuberculosis antigens & [49] \\
\hline & & & & Streptomyces griseus & Trypsin & {$[50]$} \\
\hline & Nocardia & Efficient secretion & {$[48]$} & Nocardia lactamdurans & Lysine-6-aminotransferase & [51] \\
\hline & Mycobacteria & $\begin{array}{l}\text { Posttranslational } \\
\text { modifications }\end{array}$ & {$[52]$} & $\begin{array}{l}\text { Mycobacterium } \\
\text { smegmatis }\end{array}$ & Hsp65-hlL-2 fusion protein & {$[53]$} \\
\hline & & & & & Mycobacterial proteins & {$[54]$} \\
\hline & $\begin{array}{l}\text { Coryneform } \\
\text { bacteria }\end{array}$ & $\begin{array}{l}\text { High-level production } \\
\text { and secretion; GRAS }\end{array}$ & {$[48,55]$} & $\begin{array}{l}\text { Corynebacterium } \\
\text { glutamicum }\end{array}$ & Protein-glutaminase & {$[56]$} \\
\hline & & & & $\begin{array}{l}\text { Corynebacterium } \\
\text { ammoniagenes }\end{array}$ & Pro-transglutaminase & {$[57]$} \\
\hline & & & & $\begin{array}{l}\text { Brevibacterium } \\
\text { lactofermentum }\end{array}$ & Cellulases & {$[58]$} \\
\hline \multirow[t]{10}{*}{ Firmicutes } & Bacilli & $\begin{array}{l}\text { High-level production } \\
\text { and secretion }\end{array}$ & {$[59-64]$} & Bacillus subtilis & $\beta$-Galactosidase & {$[65]$} \\
\hline & & & & Bacillus brevis & Disulfide isomerase & {$[66,67]$} \\
\hline & & & & Bacillus megaterium & Antibodies & {$[68]$} \\
\hline & & & & Bacillus licheniformis & Subtilisin & {$[69]$} \\
\hline & & & & $\begin{array}{c}\text { Bacillus } \\
\text { amyloliquefaciens }\end{array}$ & Amylases & [70] \\
\hline & $\begin{array}{l}\text { Lactic acid } \\
\text { bacteria }\end{array}$ & Secretion; GRAS & {$[22-24,71]$} & Lactococcus lactis & $\begin{array}{l}\text { Fibronectin-binding protein A, } \\
\text { internalin A, GroEL }\end{array}$ & {$[72,73]$} \\
\hline & & & & Lactobacillus plantarum & $\beta$-Galactosidase & [74] \\
\hline & & & & Lactobacillus casei & $\begin{array}{l}\text { VP2-VP3 fusion protein of infectious } \\
\text { pancreatic necrosis virus }\end{array}$ & {$[75]$} \\
\hline & & & & Lactobacillus reuteri & Pediocin PA-1 & {$[76]$} \\
\hline & & & & Lactobacillus gasseri & CC chemokines & [77] \\
\hline
\end{tabular}

${ }^{\mathrm{a} G e n e r i c ~ r e v i e w s ~ a b o u t ~ t h e ~ b i o l o g i c a l ~ p l a t f o r m ~ o r ~ a b o u t ~ s p e c i f i c ~ t o o l s ~ f o r ~ p r o t e i n ~ p r o d u c t i o n . ~}$

Towards a progressively more competitive biological synthesis by microbes [78] and assisted by expanding systems metabolic engineering and synthetic biology tools [79], industrial biotechnology should desirably find within the prokaryotic world, a growing spectrum of alternatives to eukaryotic cell factories, that apart from easy and cost-effective cultivation provide unexpectedly high metabolic versatility and biosafety of their 
protein-based products. In some cases and at a large extent, it is solving some of the main issues posed by $E$. coli as traditional producer or recombinant proteins.

\section{Competing interests}

The authors declare that they have no competing interests.

\section{Acknowledgments}

We are indebted to MINECO (BFU2010-17450), AGAUR (2009SGR-0108) and CIBER de Bioingeniería, Biomateriales y Nanomedicina (CIBER-BBN, Spain) for funding our research on protein-based therapeutics and the Protein Production Platform (PPP). CIBER-BBN is an initiative funded by the VI National R\&D\&i Plan 2008-2011, Iniciativa Ingenio 2010, Consolider Program, CIBER Actions and financed by the Instituto de Salud Carlos III with assistance from the European Regional Development Fund. AV received an ICREA ACADEMIA award.

\section{Author details}

'Institut de Biotecnologia i de Biomedicina, Universitat Autònoma de Barcelona, Bellaterra 08193 Barcelona, Spain. ${ }^{2}$ CIBER de Bioingeniería, Biomateriales y Nanomedicina (CIBER-BBN), Bellaterra 08193 Barcelona, Spain. ${ }^{3}$ Departament de Genètica i de Microbiologia, Universitat Autònoma de Barcelona, Bellaterra 08193 Barcelona, Spain.

Received: 29 October 2013 Accepted: 30 October 2013

Published: 18 November 2013

\section{References}

1. Sorensen HP: Towards universal systems for recombinant gene expression. Microb Cell Fact 2010, 9:27.

2. Ferrer-Miralles N, Domingo-Espin J, Corchero JL, Vazquez E, Villaverde A: Microbial factories for recombinant pharmaceuticals. Microb Cell Fact 2009, 8:17

3. Ward OP: Production of recombinant proteins by filamentous fungi. Biotechnol Adv 2012, 30:1119-1139.

4. Arya R, Bhattacharya A, Saini KS: Dictyostelium discoideum-a promising expression system for the production of eukaryotic proteins. FASEB $J$ 2008, 22:4055-4066.

5. Decker EL, Reski R: Moss bioreactors producing improved biopharmaceuticals. Curr Opin Biotechnol 2007, 18:393-398.

6. Potvin G, Zhang Z: Strategies for high-level recombinant protein expression in transgenic microalgae: a review. Biotechnol Adv 2010, 28:910-918

7. LEXSY Biosafety Status. 2013. http://www.jenabioscience.com/cms/en/1/ browse/1879_biosafety.html . 2013 Ref Type: Electronic Citation.

8. Porro D, Gasser B, Fossati T, Maurer M, Branduardi P, Sauer M, et al: Production of recombinant proteins and metabolites in yeasts: when are these systems better than bacterial production systems? Appl Microbiol Biotechnol 2011, 89:939-948.

9. Corchero JL, Gasser B, Resina D, Smith W, Parrilli E, Vazquez F, et al: Unconventional microbial systems for the cost-efficient production of high-quality protein therapeutics. Biotechnol Adv 2013, 31:140-153.

10. Mustalahti E, Saloheimo M, Joensuu JJ: Intracellular protein production in Trichoderma reesei (Hypocrea jecorina) with hydrophobin fusion technology. N Biotechnol 2011, 30:262-268.

11. Idiris A, Tohda H, Kumagai H, Takegawa K: Engineering of protein secretion in yeast: strategies and impact on protein production. Appl Microbiol Biotechnol 2010, 86:403-417.

12. Decker EL, Reski R: Current achievements in the production of complex biopharmaceuticals with moss bioreactors. Bioprocess Biosyst Eng 2008, 31:3-9.

13. Gerngross TU: Advances in the production of human therapeutic proteins in yeasts and filamentous fungi. Nat Biotechnol 2004, 22:1409-1414.

14. Spolaore P, Joannis-Cassan C, Duran E, Isambert A: Commercial applications of microalgae. J Biosci Bioeng 2006, 101:87-96.

15. Rme-Vega TC, Lim DK, Timmins M, Vernen F, Li Y, Schenk PM: Microalgal biofactories: a promising approach towards sustainable omega-3 fatty acid production. Microb Cell Fact 2012, 11:96.

16. Hempel F, Bozarth AS, Lindenkamp N, Klingl A, Zauner S, Linne U, et al: Microalgae as bioreactors for bioplastic production. Microb Cell Fact 2011, 10:81.
17. Specht E, Miyake-Stoner S, Mayfield S: Micro-algae come of age as a platform for recombinant protein production. Biotechnol Lett 2010, 32:1373-1383.

18. Rhee SJ, Lee JE, Lee CH: Importance of lactic acid bacteria in Asian fermented foods. Microb Cell Fact 2011, 10(1):S5.

19. De Vos WM: Systems solutions by lactic acid bacteria: from paradigms to practice. Microb Cell Fact 2011, 10(1):S2.

20. Arendt EK, Moroni A, Zannini E: Medical nutrition therapy: use of sourdough lactic acid bacteria as a cell factory for delivering functional biomolecules and food ingredients in gluten free bread. Microb Cell Fact 2011, 10(1):S15.

21. Teusink B, Bachmann H, Molenaar D: Systems biology of lactic acid bacteria: a critical review. Microb Cell Fact 2011, 10(1):S11.

22. Garcia-Fruitos E: Lactic Acid Bacteria: a promising alternative for recombinant protein production. Microb Cell Fact 2012, 11:157.

23. Peterbauer $C$, Maischberger $T$, Haltrich D: Food-grade gene expression in lactic acid bacteria. Biotechnol J 2011, 6:1147-1161.

24. Morello E, Bermudez-Humaran LG, Llull D, Sole V, Miraglio N, Langella P, et al: Lactococcus lactis, an efficient cell factory for recombinant protein production and secretion. J Mol Microbiol Biotechnol 2008, 14:48-58.

25. Pontes DS, de Azevedo MS, Chatel JM, Langella P, Azevedo V, Miyoshi A: Lactococcus lactis as a live vector: heterologous protein production and DNA delivery systems. Protein Expr Purif 2011, 79:165-175.

26. Daniel C, Roussel Y, Kleerebezem M, Pot B: Recombinant lactic acid bacteria as mucosal biotherapeutic agents. Trends Biotechnol 2011 29:499-508.

27. Hu S, Kong J, Sun Z, Han L, Kong W, Yang P: Heterologous protein display on the cell surface of lactic acid bacteria mediated by the S-layer protein. Microb Cell Fact 2011, 10:86.

28. Bermudez-Humaran LG, Kharrat P, Chatel JM, Langella P: Lactococci and lactobacilli as mucosal delivery vectors for therapeutic proteins and DNA vaccines. Microb Cell Fact 2011, 10(1):S4

29. Scavone P, Miyoshi A, Rial A, Chabalgoity A, Langella P, Azevedo V, et al: Intranasal immunisation with recombinant lactococcus lactis displaying either anchored or secreted forms of proteus mirabilis MrpA fimbrial protein confers specific immune response and induces a significant reduction of kidney bacterial colonisation in mice. Microbes Infect 2007, 9:821-828.

30. Chen R: Bacterial expression systems for recombinant protein production: E. coli and beyond. Biotechnol Adv 2012, 30:1102-1107.

31. Terpe K: Overview of tag protein fusions: from molecular and biochemical fundamentals to commercial systems. Appl Microbiol Biotechnol 2003, 60:523-533.

32. Hahn HP, von Specht BU: Secretory delivery of recombinant proteins in attenuated salmonella strains: potential and limitations of type I protein transporters. FEMS Immunol Med Microbiol 2003, 37:87-98.

33. Simon B, Nomellini J, Chiou P, Bingle W, Thornton J, Smit J, et al: Recombinant vaccines against infectious hematopoietic necrosis virus: production by the Caulobacter crescentus S-layer protein secretion system and evaluation in laboratory trials. Dis Aquat Organ 2001, 44:17-27.

34. Duncan G, Tarling CA, Bingle WH, Nomellini JF, Yamage M, Dorocicz IR, et al: Evaluation of a new system for developing particulate enzymes based on the surface (S)-layer protein (RsaA) of Caulobacter crescentus: fusion with the beta-1,4-glycanase (Cex) from the cellulolytic bacterium Cellulomonas fimi yields a robust, catalytically active product. App/ Biochem Biotechnol 2005, 127:95-110.

35. Laible PD, Scott HN, Henry L, Hanson DK: Towards higher-throughput membrane protein production for structural genomics initiatives. I Struct Funct Genomics 2004, 5:167-172.

36. Duilio A, Tutino ML, Marino G: Recombinant protein production in Antarctic Gram-negative bacteria. Methods Mol Biol 2004, 267:225-237.

37. Rippa V, Papa R, Giuliani M, Pezzella C, Parrilli E, Tutino ML, et al: Regulated recombinant protein production in the Antarctic bacterium Pseudoalteromonas haloplanktis TAC125. Methods Mol Biol 2012, 824:203-218.

38. Giuliani M, Parrilli E, Ferrer P, Baumann K, Marino C, Tutino ML: Process optimization for recombinant protein production in the psychrophilic bacterium Pseudoalteromonas haloplanktis. Process Biochem 2011, 46:953-959.

39. Vigentini I, Merico A, Tutino ML, Compagno C, Marino G: Optimization of recombinant human nerve growth factor production in the 
psychrophilic Pseudoalteromonas haloplanktis. J Biotechnol 2006, 127:141-150.

40. Miyake R, Kawamoto J, Wei YL, Kitagawa M, Kato I, Kurihara T, et al: Construction of a low-temperature protein expression system using a cold-adapted bacterium, Shewanella sp. strain Ac10, as the host. App/ Environ Microbiol 2007, 73:4849-4856.

41. Retallack DM, Jin H, Chew L: Reliable protein production in a Pseudomonas fluorescens expression system. Protein Expr Purif 2011 81:157-165.

42. Jin H, Cantin GT, Maki S, Chew LC, Resnick SM, Ngai J, et al: Soluble periplasmic production of human granulocyte colony-stimulating factor (G-CSF) in Pseudomonas fluorescens. Protein Expr Purif 2011, 78:69-77.

43. Dammeyer T, Steinwand M, Kruger SC, Dubel S, Hust M, Timmis KN: Efficient production of soluble recombinant single chain Fv fragments by a Pseudomonas putida strain KT2440 cell factory. Microb Cell Fact 2011, 10:11.

44. Krzeslak J, Braun P, Voulhoux R, Cool RH, Quax WJ: Heterologous production of Escherichia coli penicillin $\mathrm{G}$ acylase in Pseudomonas aeruginosa. J Biotechnol 2009, 142:250-258.

45. Tokunaga H, Arakawa T, Tokunaga M: Novel soluble expression technologies derived from unique properties of halophilic proteins. Appl Microbiol Biotechnol 2010, 88:1223-1231.

46. Nagayoshi C, Tokunaga $\mathrm{H}$, Hayashi A, Harazono H, Hamasaki K, Ando A, et al: Efficient expression of haloarchaeal nucleoside diphosphate kinase via strong porin promoter in moderately halophilic bacteria. Protein Pept Lett 2006, 13:611-615.

47. Anne J, Maldonado B, Van IJ, Van ML, Bernaerts K: Recombinant protein production and streptomycetes. J Biotechnol 2012, 158:159-167.

48. Liu L, Yang H, Shin HD, Li J, Du G, Chen J: Recent advances in recombinant protein expression by Corynebacterium, Brevibacterium, and Streptomyces: from transcription and translation regulation to secretion pathway selection. Appl Microbiol Biotechnol 2013, 97:9597-9608.

49. Ayala JC, Pimienta E, Rodriguez C, Anne J, Vallin C, Milanes MT, et al: Use of Strep-tag II for rapid detection and purification of Mycobacterium tuberculosis recombinant antigens secreted by Streptomyces lividans. J Microbiol Methods 2013, 94:192-198.

50. Chi WJ, Song JH, Oh EA, Park SW, Chang YK, Kim ES, et al: Medium optimization and application of an affinity column chromatography for streptomyces griseus trypsin production from the recombinant Streptomyces griseus. J Microbiol Biotechnol 2009, 19:1191-1196.

51. Chary VK, de la Fuente $J$, Leitao AL, Liras P, Martin JF: Overexpression of the lat gene in Nocardia lactamdurans from strong heterologous promoters results in very high levels of lysine-6-aminotransferase and up to two-fold increase in cephamycin C production. Appl Microbiol Biotechnol 2000, 53:282-288.

52. Connell ND: Expression systems for use in actinomycetes and related organisms. Curr Opin Biotechnol 2001, 12:446-449.

53. Guo XQ, Wei YM, Yu B: Recombinant Mycobacterium smegmatis expressing Hsp65-hlL-2 fusion protein and its influence on lymphocyte function in mice. Asian Pac J Trop Med 2012, 5:347-351.

54. Noens EE, Williams C, Anandhakrishnan M, Poulsen C, Ehebauer MT, Wilmanns M: Improved mycobacterial protein production using a Mycobacterium smegmatis groEL1DeltaC expression strain. BMC Biotechnol 2011, 11:27.

55. Srivastava P, Deb JK: Gene expression systems in corynebacteria. Protein Expr Purif 2005, 40:221-229.

56. Kikuchi Y, Itaya H, Date M, Matsui K, Wu LF: Production of Chryseobacterium proteolyticum protein-glutaminase using the twin-arginine translocation pathway in Corynebacterium glutamicum. Appl Microbiol Biotechnol 2008, 78:67-74.

57. Itaya H, Kikuchi Y: Secretion of Streptomyces mobaraensis protransglutaminase by coryneform bacteria. Appl Microbiol Biotechnol 2008, 78:621-625.

58. Paradis FW, Warren RA, Kilburn DG, Miller RC Jr: The expression of Cellulomonas fimi cellulase genes in Brevibacterium lactofermentum. Gene 1987, 61:199-206.

59. van Dijl JM, Hecker M: Bacillus subtilis: from soil bacterium to super-secreting cell factory. Microb Cell Fact 2013, 12:3.

60. Terpe K: Overview of bacterial expression systems for heterologous protein production: from molecular and biochemical fundamentals to commercial systems. Appl Microbiol Biotechnol 2006, 72:211-222.
61. Westers L, Westers H, Quax WJ: Bacillus subtilis as cell factory for pharmaceutical proteins: a biotechnological approach to optimize the host organism. Biochim Biophys Acta 2004, 1694:299-310.

62. Pohl S, Harwood CR: Heterologous protein secretion by bacillus species from the cradle to the grave. Adv Appl Microbiol 2010, 73:1-25.

63. Biedendieck R, Bunk B, Furch T, Franco-Lara E, Jahn M, Jahn D: Systems biology of recombinant protein production in bacillus megaterium. Adv Biochem Eng Biotechnol 2010, 120:133-161.

64. Schallmey M, Singh A, Ward OP: Developments in the use of Bacillus species for industrial production. Can J Microbiol 2004, 50:1-17.

65. Yang M, Zhang W, Ji S, Cao P, Chen Y, Zhao X: Generation of an artificial double promoter for protein expression in Bacillus subtilis through a promoter trap system. PLoS One 2013, 8:e56321.

66. Kajino T, Ohto C, Muramatsu M, Obata S, Udaka S, Yamada Y, et al: A protein disulfide isomerase gene fusion expression system that increases the extracellular productivity of Bacillus brevis. Appl Environ Microbiol 2000, 66:638-642.

67. Kajino T, Kato K, Miyazaki C, Asami O, Hirai M, Yamada Y, et al: Isolation of a protease-deficient mutant of Bacillus brevis and efficient secretion of a fungal protein disulfide isomerase by the mutant. J Biosci Bioeng 1999, 87:37-42.

68. David F, Steinwand M, Hust M, Bohle K, Ross A, Dubel S, et al: Antibody production in Bacillus megaterium: strategies and physiological implications of scaling from micro titer plates to industrial bioreactors. Biotechnol J 2011, 6:1516-1531.

69. Toyokawa Y, Takahara H, Reungsang A, Fukuta M, Hachimine Y, Tachibana S, et al: Purification and characterization of a halotolerant serine proteinase from thermotolerant Bacillus licheniformis RKK-04 isolated from Thai fish sauce. Appl Microbiol Biotechnol 2010, 86:1867-1875.

70. Deb P, Talukdar SA, Mohsina K, Sarker PK, Sayem SA: Production and partial characterization of extracellular amylase enzyme from P-001. Springerplus 2013, 2:154.

71. Le LY, Azevedo V, Oliveira SC, Freitas DA, Miyoshi A, Bermudez-Humaran LG, et al: Protein secretion in Lactococcus lactis: an efficient way to increase the overall heterologous protein production. Microb Cell Fact 2005, 4:2

72. Innocentin S, Guimaraes V, Miyoshi A, Azevedo V, Langella P, Chatel JM, et al: Lactococcus lactis expressing either Staphylococcus aureus fibronectin-binding protein A or Listeria monocytogenes internalin A can efficiently internalize and deliver DNA in human epithelial cells. Appl Environ Microbiol 2009, 75:4870-4878.

73. Miyoshi A, Bermudez-Humaran LG, Ribeiro LA, Le LY, Oliveira SC, Langella P, et al: Heterologous expression of Brucella abortus GroEL heat-shock protein in Lactococcus lactis. Microb Cell Fact 2006, 5:14.

74. Nguyen TT, Nguyen HA, Arreola SL, Mlynek G, Djinovic-Carugo K, Mathiesen $\mathrm{G}$, et al: Homodimeric beta-galactosidase from Lactobacillus delbrueckii subsp. bulgaricus DSM 20081: expression in Lactobacillus plantarum and biochemical characterization J Agric Food Chem 2012, 60:1713-1721.

75. Zhao LL, Liu M, Ge JW, Qiao XY, Li YJ, Liu DQ: Expression of infectious pancreatic necrosis virus (IPNV) VP2-VP3 fusion protein in Lactobacillus casei and immunogenicity in rainbow trouts. Vaccine 2012, 30:1823-1829.

76. Eom JE, Moon SK, Moon GS: Heterologous production of pediocin PA-1 in Lactobacillus reuteri. J Microbiol Biotechnol 2010, 20:1215-1218.

77. Damelin LH, Mavri-Damelin D, Klaenhammer TR, Tiemessen CT: Plasmid transduction using bacteriophage Phi(adh) for expression of CC chemokines by Lactobacillus gasseri ADH. Appl Environ Microbiol 2010, 76:3878-3885.

78. Chen GQ: New challenges and opportunities for industrial biotechnology. Microb Cell Fact 2012, 11:111.

79. Lee SY, Mattanovich D, Villaverde A: Systems metabolic engineering, industrial biotechnology and microbial cell factories. Microb Cell Fact 2012, 11:156.

\section{doi:10.1186/1475-2859-12-113}

Cite this article as: Ferrer-Miralles and Villaverde: Bacterial cell factories for recombinant protein production; expanding the catalogue. Microbial Cell Factories 2013 12:113. 